BMJ Open Diabetes

Research \& Care

\title{
Healthy Eating and Active Lifestyles for Diabetes (HEAL-D), a culturally tailored self-management education and support program for type 2 diabetes in black- British adults: a randomized controlled feasibility trial
}

Louise M Goff (D , , ${ }^{1}$ Carol Rivas, ${ }^{2}$ Amanda Moore, ${ }^{1}$ Nicholas Beckley-Hoelscher, ${ }^{3}$ Fiona Reid, ${ }^{3}$ Seeromanie Harding ${ }^{1}$

\section{ABSTRACT}

Introduction Black-British communities are

disproportionately affected by type 2 diabetes (T2D).

Structured education programs are a core component of

T2D healthcare but they are less successful in people from

minority ethnic groups. Culturally tailored T2D education has demonstrated greater benefits than usual care. The aim of our study was to evaluate acceptability, fidelity and trial feasibility of the Healthy Eating and Active Lifestyles for Diabetes ('HEAL-D') culturally tailored T2D self-management education and support (DSMES) program.

Research design and methods A mixed-methods randomized controlled feasibility trial in black-British adults with T2D was conducted. Participants were assigned to control (usual care) or intervention (HEAL-D; 7 sessions, 14 hours of group-based culturally tailored diet and lifestyle education, behavior change support and supervised physical activity), in a ratio of 1:1. Primary outcomes were recruitment and retention rates, intervention attendance and completion. Fidelity was assessed through observations and qualitative evaluation was undertaken with participants and educators.

Results 102 patients responded to invitation letters ( $n=1335)$; 63 were randomized but 8 were subsequently deemed ineligible due to high baseline glycosylated hemoglogin ( $\mathrm{HbA1c}$ ) requiring intensive medical management or missing baseline $\mathrm{HbA} 1 \mathrm{c}$ measurement. Of the remaining 55 participants (27 intervention, 28 control), 69\% were female, $47 \%$ were of African and $51 \%$ were of Caribbean ethnicity. $93 \%$ completed the trial, providing end point data. Intervention attendance was high; $85 \%$ completed the program (attendance at $\geq 5$ sessions), and $74 \%$ attended $\geq 6$ sessions. The intervention was delivered with acceptable fidelity, although the qualitative evaluations identified some areas of structure and format in need of refinement.

Conclusions We have shown it is feasible to recruit and randomize black-British adults with T2D to a trial of a culturally tailored DSMES program. We have shown the intervention is highly acceptable for both patients and healthcare providers. A future trial should assess clinical and cost-effectiveness of HEAL-D.

Trial registration number NCT03531177.

\section{SIGNIFICANCE OF THIS STUDY \\ WHAT IS ALREADY KNOWN ABOUT THIS SUBJECT? \\ $\Rightarrow$ Black-British communities are disproportionately affected by type 2 diabetes. \\ $\Rightarrow$ Diabetes structured education programs are less successful in people from minority ethnic groups. \\ $\Rightarrow$ Culturally tailored diabetes education has demon- strated greater improvements in diabetes control and knowledge than usual care, and the benefits are maintained long-term but there are no such pro- grams for black-British adults.}

\section{WHAT ARE THE NEW FINDINGS?}

$\Rightarrow$ We have shown that it is feasible to recruit and randomize black-British adults with type 2 diabetes to a trial of a culturally tailored diabetes selfmanagement education and support program.

$\Rightarrow$ We have shown a culturally tailored diabetes selfmanagement education and support program to be highly acceptable for both patients and healthcare providers.

\section{HOW MIGHT THESE RESULTS CHANGE THE FOCUS OF RESEARCH OR CLINICAL PRACTICE?}

$\Rightarrow$ Improving the cultural sensitivity of diabetes selfmanagement education is an important means by which to improve the management of type 2 diabetes in minority ethnic groups.

$\Rightarrow$ The evaluation of the real-world delivery of the Healthy Eating and Active Lifestyles for Diabetes culturally tailored diabetes self-management education and support program has shown it to be highly acceptable to both black-British adults living with type 2 diabetes and healthcare professionals involved in their management.

\section{INTRODUCTION}

In the UK, as in other upper-middle-income and high-income countries, minority ethnic 
groups are disproportionately affected by type 2 diabetes (T2D). ${ }^{1}$ In black-British communities, T2D is three times more prevalent than among white-Europeans ${ }^{2}$ and poorer outcomes are evident. ${ }^{34}$

Supporting people living with T2D to make healthy diet and lifestyle changes forms the cornerstone of management. ${ }^{56}$ Black-British adults are recognised to participate in relatively low levels of physical activity ${ }^{7}$ and consume diets high in carbohydrate and salt. ${ }^{8}$ Self-management and lifestyle change is challenging, adherence is a major issue, with only a minority of patients achieving their treatment goals. ${ }^{9}$ Several factors influence adherence, including knowledge gaps, personal and cultural beliefs as well as barriers between patients and healthcare practitioners relating to communication and access to, and quality of, care and education. ${ }^{10}$

Diabetes self-management education and support (DSMES) has been shown to enhance self-management. ${ }^{11}$ Management guidelines (eg, UK National Institute for Health and Care Excellence) recommend provision of structured education programs to support the development of self-management skills. ${ }^{12}$ These programs are effective at improving clinical outcomes and well-being. However, they are mostly based on generic advice that is not sensitive diverse cultures and there is evidence that they are less successful in people from minority ethnic groups. ${ }^{1314}$ This is often attributed to healthcare practitioners lacking cultural knowledge and awareness, and a failure to account for cultural beliefs and practices in generic education programs. ${ }^{15}$ Culturally tailored DSMES programs that are responsive to the health beliefs and cultural practices of minority ethnic groups have demonstrated greater improvements in T2D control and knowledge than usual care. ${ }^{15}$ However, to date, culturally tailored DSMES interventions for communities of black-African ancestry have largely been based in the USA, and may not translate to UK healthcare structures or black-British communities, whose cultural needs may be different. ${ }^{16}$ To address this gap, we developed an evidence-based, culturally tailored DSMES program for black-British communities, called Healthy Eating and Active Lifestyles for Diabetes (HEAL-D). ${ }^{17}$

Prior to a definitive evaluation, we undertook a mixed methods feasibility trial to evaluate key considerations, namely intervention acceptability, fidelity and trial feasibility. Specific objectives were to determine:

- the proportion and characteristics of black-British adults living with T2D who are willing to participate in a randomized controlled trial (RCT) of a culturally tailored DSMES program (ie, recruitment and retention rates);

- feasibility of data collection for potential primary and secondary outcomes;

- estimates of SD for potential primary outcomes, to enable trial sample size calculations;

- estimates of the change in glycosylated hemoglogin (HbAlc) to provide a signal of efficacy;

- the rates of attendance and intervention acceptability; if a culturally tailored DSMES program can be delivered with fidelity.

\section{PARTICIPANTS AND METHODS}

\section{Trial design}

A single-center, parallel-group, RCT design was conducted, the protocol has been published previously. ${ }^{18}$

\section{Participants}

Recruitment was open April-October 2018. In the London Boroughs of Lambeth and Southwark, primary care database searches identified potential participants and letters of invitation were sent. Additionally, primary and intermediate care practitioners referred participants that they identified through their practice, and posters/ flyers were distributed in practices for self-referral. Participants from the preceding intervention development project were invited to participate but, due to their prior involvement, they were allocated to receive HEAL-D and were not included in the randomization process.

Eligible participants were of self-declared blackBritish, African or Caribbean ethnicity; aged $\geq 18$ years; had a clinical diagnosis of T2D; able to communicate in English and suitable for general diet and lifestyle advice, and group-based education (ie, no complex diet needs such as chronic kidney disease, or complex learning needs; suitability judged by the referring healthcare practitioner). Exclusion criteria included pregnancy and complex clinical needs. Following recruitment, an additional criterion was added (October 2018), HbAlc $<86 \mathrm{mmol} / \mathrm{mol}$, after a proportion of participants were found to have high baseline values that needed intensive medical management.

\section{Randomization}

Individual participants were randomized (1:1 ratio, without blocking) to intervention (culturally tailored DSMES program plus usual care) or control (usual care alone), using sealed envelopes. The research assistant performed randomization at the baseline visit after gaining consent before data collection. The research assistant was aware of allocation but the nurses conducting the biomedical assessments were not. Participants from the intervention development study were recruited but not randomized or included in the main outcome data due to issues of contamination; they were included in the qualitative evaluation of intervention acceptability.

\section{Sample size}

A pragmatic sample of 60 randomized participants, 30 in each arm, was anticipated to be sufficient to evaluate the program, allowing for $10 \%$ drop-out/noncompletion. Delays in research governance approval processes and restrictions preventing extension of the study end, required a sample size reduction from that of the published protocol to $\mathrm{n}=60$; this amendment was approved prior to trial commencement. 


\section{The intervention}

The intervention was developed through a co-design project; ${ }^{17}$ HEAL-D, consisted of 14 hours of face-to-face, group-based culturally tailored education, behavior change support and participatory physical activity. Seven sessions, each 2 hours, were delivered by a lay educator of black-British ethnicity and a diabetes specialist registered dietitian (no specific ethnicity). Physical activity classes, delivered by exercise instructors trained in rehabilitation exercise, were included in five sessions; these included resistance (eg, circuit training and resistance band training) and cardiorespiratory (eg, walking group, dance aerobics and Zumba) exercises. Sessions were scheduled for daytime, evening and weekend delivery, using a weekly or fortnightly schedule, with participants choosing the program location and timing that best suited their needs. 'Flexible attendance' allowed participants to switch between programs where needed/desired, for example, missed sessions. The sessions were delivered in community venues such as church halls or community centres, aiming for group sizes of 8-12 participants. The sessions followed an evidence-based curriculum, focusing on four specific diet and lifestyle goals:

I. Carbohydrates: limit portion sizes.

II. Physical activity: participate in $30 \mathrm{~min}$ of moderate to vigorous physical activity daily and strength training twice a week.

III. Body weight: lose $5 \%-10 \%$ body weight if overweight/obese or maintain a healthy weight.

IV. Cardiovascular risk: limit saturated fat and salt intake.

Culturally tailored materials, including information booklets, games and videos were developed. A range of behavior change techniques (BCTs) were used (online supplemental table S1), selected from analysis of the qualitative data collected in the co-design study, which identified key barriers relating to the behavioral goals of the intervention (full details of the intervention ${ }^{17}$ and choice of BCTs ${ }^{19}$ have been published).

An educator delivery manual was developed, which detailed the structure and delivery of the sessions. Delivery of specific sections of the sessions were designated to the different educator roles, whereby the dietitian mainly led on education/information and discussion-based sections and the lay educator led on interactive games and tasks.

The educators received 8 hours of formal training, delivered by the lead researcher (LMG); both educator roles received training on the learning objectives of the sessions, how to deliver the different components of the sessions and the theory and delivery of the BCTs. In addition, the lay educators received training on T2D and principles of self-management while the dietitian educators received training on important cultural beliefs and practices relevant to the intervention.

Lay educators were recruited via a range of channels, including the Diabetes UK 'Community Champion' initiative, which trains members of ethnic minority communities to raise $\mathrm{T} 2 \mathrm{D}$ awareness among their communities (eg, risk screening roadshows), and through the research teams' networks. Lay educators were considered eligible if they were of black-British ethnicity, with good command of written and spoken English, an interest in health and/or T2D, effective presentation skills, confident at communicating and working with the public and having rights to work in the UK. Dietitian educators were recruited from clinical service provision in south London.

Usual care was determined by the participants' medical team, typically in primary care, and was not actively influenced by the research team. Both arms received usual care; there was no other intervention provided to the control arm. Medical management of diabetes, blood pressure and lipids were undertaken by the participants' primary care physician throughout the study.

\section{Outcome measures}

Measures were taken at baseline (randomization) and 6-8 months postrandomization; the majority started the intervention within 2-4 weeks of randomization, however, for a small number this was delayed by 2-4 weeks due to program scheduling and locations. In all cases, follow-up was scheduled for 6 months after starting the intervention. All outcome data, other than intervention acceptability and fidelity, were collected in a 2-hour study visit at the Clinical Research Facility at Guy's and St Thomas' NHS Foundation Trust, London, UK.

\section{Biomedical measures}

HbA1c, blood lipids, blood pressure, weight, height, body mass index and waist circumference were measured with the participant fasting and wearing light clothing (shoes removed). Standard operating procedures ensured quality and consistency. Samples were drawn from a venous sample and assayed locally in the accredited hospital laboratory.

\section{Patient report outcome measures}

The following questionnaire measures were completed: Perceived Diabetes \& Dietary Competence ${ }^{20}$ (dietary competence) and International Physical Activity Questionnaireshort form ${ }^{21}$ (physical activity) to assess lifestyle changes; Short Diabetes Knowledge Instrument ${ }^{22}$ (diabetes knowledge), Diabetes Empowerment Scale Short Form ${ }^{23}$ (empowerment) and Multidimensional Scale of Perceived Social Support $^{24}$ (social support) to assess potential intervention mechanisms and EuroQol EQ5D-3L visual analog scale ${ }^{25}$ (quality of life) and 5-item Problem Areas In Diabetes scale $^{26}$ (diabetes distress) as potential intervention outcomes.

\section{Acceptability and fidelity of the intervention}

Intervention participants participated in focus groups, conducted after completion of the program, to evaluate overall intervention acceptability as well as key components of format, structure and content. A sample of sessions were observed using a bespoke checklist to assess fidelity of delivery and to identify any refinements 
that were needed. Selection of sessions for fidelity observations aimed to ensure each session within the course was observed, to determine adherence to the delivery manual and at least half of the course sessions were observed more than once and with different educator pairings, to determine variability in adherence to the delivery manual.

Adherence to intervention elements was scored as: occurrence ( 2 points), attempted occurrence (1 point) or non-occurrence (0 points); means were calculated. Adherence to scheduled times was calculated as per cent actual/recommended time. Educators were interviewed after delivering the intervention to evaluate acceptability from an educator perspective and to identify any refinements that were needed. All focus groups and interviews were digitally recorded and transcribed verbatim. The transcripts data were analysed by one member of the research team (CR) for descriptive themes using thematic content analysis with data managed using NVivo V.10 (QSR International, 2021) and with themes discussed by the team. As themes were descriptive process considerations, extensive double coding was deemed unnecessary. However, all extracts for each theme were read by a team member (LMG) against each theme name, for validation.

\section{Statistical analysis}

Descriptive analyses are presented. Recruitment rates were calculated in two ways: the number randomized as a percentage of people who were sent letters of invitation and the number randomized as a percentage of people who expressed an interest in participation. Intervention adherence was assessed using attendance records; completion of HEAL-D was defined as attendance at $\geq 5$ out of 7 sessions. Retention was calculated as the number of participants who attended the end point visit as a percentage of those recruited. Data completion was calculated as the proportion of participants who had paired baseline and end point data. These rates were evaluated by sex, ethnicity, age group and employment status. Rates are expressed as number (\%), and clinical and patient-reported data are presented as mean (SD), with $95 \%$ CIs.

For variables that may form primary and secondary outcomes in a definitive trial, the mean at baseline and follow-up has been calculated, as well as the mean of the change from baseline to follow-up (change score). To account for regression to the mean effects, the difference in change scores between treatment groups was adjusted for the baseline values. The adjusted mean difference in change score has been presented with $95 \%$ CI, providing signal of efficacy estimates. In line with the Consolidated Standards of Reporting Trials extension for pilot studies and because we were not formally powered to detect between-group differences in outcomes, differences have not been tested for significance. Analyses were conducted using Stata V.15 (StataCorp, 2017).
RESULTS

\section{Participants and characteristics}

A total of 102 black-British adults with T2D expressed an interest in participating in response to invitation letters sent from database searches of 11 primary and intermediate care practices (1335 letters sent). Of these, 63 consented and were randomized. A further 14 participants from the intervention development study participated and were allocated (non-randomized) to the intervention. Following baseline assessment of HbAlc, 5 randomized participants $(n=3$ intervention, $n=2$ control) were found to have HbAlc levels $\geq 86 \mathrm{mmol} / \mathrm{mol}$ $(10 \%)$, requiring referral for intensive medical management; these participants were considered ineligible and excluded from data analysis, although they continued to receive the intervention and, due to the group-based nature of the intervention acceptability evaluations, were included in the qualitative data collection (figure 1). A further 3 participants did not have HbA1c measured at baseline, leaving 55 randomized participants in the main quantitative analyses.

Of the 55 participants, $69 \%$ were female, there was an equal mix of participants of direct African versus Caribbean ethnicity, $73 \%$ were first-generation migrants (born outside UK), $42 \%$ were in receipt of welfare benefits and $38 \%$ were in paid work (table 1 ).

Six HEAL-D courses were delivered between April and December 2018 in five different community venues in the London Boroughs of Lambeth and Southwark. Four dietitian (two of black-British and two of whiteBritish ethnicity) and four lay educators (all black-British ethnicity) delivered the courses, with all sessions within a course delivered by the same educator pairing. The group sizes ranged from 2 to 10 , with an average of six participants per course.

\section{Trial recruitment and retention}

The recruitment rate was calculated based on the number of eligible randomized participants $(n=55)$ in relation to the number of invitation letters sent $(\mathrm{n}=1335)(4 \%(95 \%$ CI 3 to 5)) and in relation to the number who expressed interest in response to the invitation letters $(\mathrm{n}=102)$ (54\% (95\% CI 44 to 64$)$ ).

Fifty-one of the 55 randomized participants completed the trial, giving a retention rate of $93 \%$ (95\% CI 82 to 98); retention rates were equal across intervention and control arms (online supplemental table S2) and there were no appreciable differences seen by sex, ethnicity, age group and employment status (online supplemental table S2). Most withdrawals were due to missing the end point visits, in which two participants were sick or travelling and two participants could not be contacted.

\section{Intervention attendance}

Attendance at the intervention was high: 85\% (23/27) completed the program (attendance at $\geq 5$ sessions), and $74 \%(20 / 27)$ attended $\geq 6$ sessions. No appreciable differences in attendance were seen in relation to sex, ethnicity, 


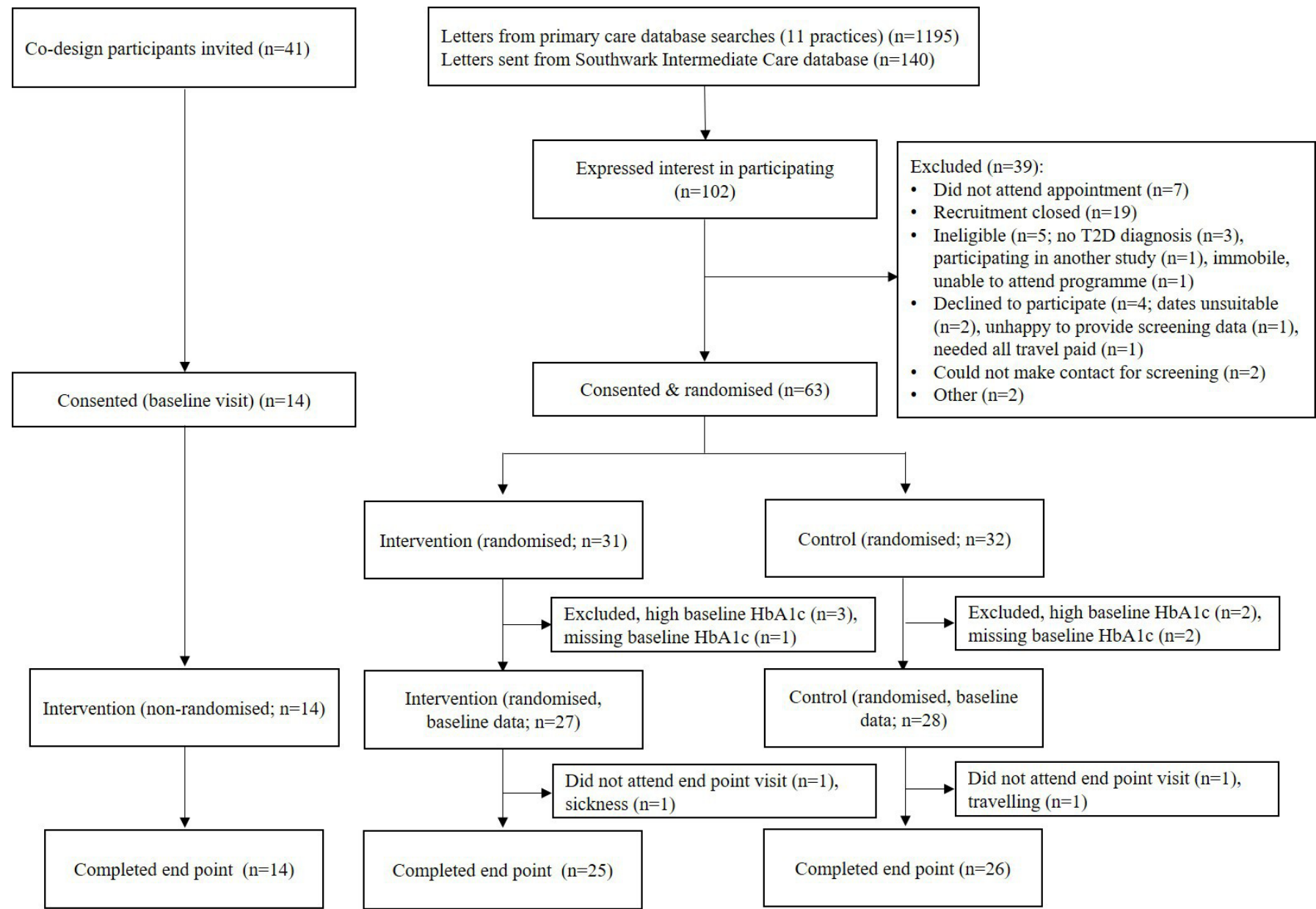

Figure 1 Consolidated Standards of Reporting Trials diagram. Flow of participants through the Healthy Eating and Active Lifestyles for Diabetes feasibility trial. HbA1c, glycosylated hemoglogin; T2D, type 2 diabetes.

age group and employment status (online supplemental table S3). A third of participants (10/27) used 'flexible attendance', switching between courses, thus enabling them to attend more sessions/complete the program.

\section{Trial outcome data and estimates of sample size}

Of the 55 participants who entered the trial, 93\% ( $\mathrm{n}=51)$ had complete outcome data for HbAlc, weight and lipids. Each patient-reported outcome variable was complete for at least $85 \%(n=47)$ of participants (online supplemental table S4 and S5).

Table 2 shows the clinical and patient report outcome measures, by arm and timepoint. Mean baseline HbAlc was 60.6 (SD 11.4) and 59.1 (SD 11.2) $\mathrm{mmol} / \mathrm{mol}$ in the intervention and control groups, respectively. Adjusted mean difference in HbAlc change scores between intervention and control was $-2.8(95 \% \mathrm{CI}-9.5$ to 3.9$) \mathrm{mmol} /$ mol. The primary outcome in a future definitive trial would be $\mathrm{HbAlc}$, as the main clinical measure used to assess overall glycemic control and a predictor of longterm complications. To inform the sample size calculation for a future definitive trial, we analyzed the Pearson's correlation coefficient between baseline and end point HbA1c, which is estimated to be 0.672 , as well as the SD of HbAlc values by treatment group (table 2).
Self-reported physical activity is shown in table 3. At baseline, $48 \%$ and $43 \%$ of participants in the intervention arm and $40 \%$ and $52 \%$ of participants in the control arm rated their physical activity as low and moderate, respectively. At end point, the proportion of intervention participants who rated their physical activity as low decreased by 17 percentage points and moderate increased by 25 percentage points, while in the control group low physical activity dropped by 4 percentage points and moderate increased by 4 percentage points.

\section{Fidelity and acceptability}

Thirteen sessions were observed, no session was observed by more than one person (online supplemental table S5). Focus groups were conducted with participants from four (out of six) courses, and interviews were conducted with all educators, to understand the extent to which components of the program were operationalized as intended, how acceptable and effective they were and what refinements were needed. Online supplemental table S6 provides a summary of the findings, with illustrative quotes from participants and educators. 
Table 1 Sociodemographic characteristics of study participants

\begin{tabular}{|c|c|c|c|}
\hline & $\begin{array}{l}\text { Overall } \\
(n=55)\end{array}$ & $\begin{array}{l}\text { Intervention } \\
\text { group } \\
(\mathrm{n}=27)\end{array}$ & $\begin{array}{l}\text { Control } \\
\text { group } \\
(n=28)\end{array}$ \\
\hline \multicolumn{4}{|l|}{ Sex, n (\%) } \\
\hline Female & $38(69)$ & $18(67)$ & $20(71)$ \\
\hline Male & $17(31)$ & $9(33)$ & $8(29)$ \\
\hline \multicolumn{4}{|l|}{ Ethnicity, n (\%) } \\
\hline African & $26(47)$ & $10(37)$ & $16(57)$ \\
\hline Caribbean & $28(51)$ & $16(59)$ & $12(43)$ \\
\hline $\begin{array}{l}\text { Mixed white and } \\
\text { black African }\end{array}$ & $1(2)$ & $1(4)$ & $0(0)$ \\
\hline \multicolumn{4}{|l|}{ Age (years), n (\%) } \\
\hline$<45$ & $3(5)$ & $1(4)$ & $2(7)$ \\
\hline $45-55$ & $16(29)$ & $9(33)$ & $7(25)$ \\
\hline $55-64$ & $20(36)$ & $8(30)$ & $12(43)$ \\
\hline $65-74$ & $12(22)$ & $6(22)$ & $6(21)$ \\
\hline$\geq 75$ & $4(7)$ & $3(11)$ & $1(4)$ \\
\hline \multicolumn{4}{|c|}{ Employment status, n (\%) } \\
\hline $\begin{array}{l}\text { Paid/Self- } \\
\text { employed }\end{array}$ & $21(38)$ & $11(41)$ & $10(36)$ \\
\hline Voluntary & $2(4)$ & $0(0)$ & $2(7)$ \\
\hline Unemployed & $10(18)$ & $4(15)$ & $6(21)$ \\
\hline Student & $0(0)$ & $0(0)$ & $0(0)$ \\
\hline $\begin{array}{l}\text { Housewife/ } \\
\text { Husband }\end{array}$ & $3(5)$ & $2(7)$ & $1(4)$ \\
\hline Retired & $19(35)$ & $10(37)$ & $9(32)$ \\
\hline \multicolumn{4}{|c|}{ Generational status, n (\%) } \\
\hline First generation & $40(73)$ & $21(78)$ & $19(68)$ \\
\hline $\begin{array}{l}\text { Second } \\
\text { generation }\end{array}$ & $13(24)$ & $5(19)$ & $8(29)$ \\
\hline Missing & $2(4)$ & $1(4)$ & $1(4)$ \\
\hline \multicolumn{4}{|c|}{ Educational attainment, n (\%) } \\
\hline Primary & $0(0)$ & $0(0)$ & $0(0)$ \\
\hline Secondary 16 & $10(18)$ & $7(26)$ & $3(11)$ \\
\hline Secondary 18 & $14(25)$ & $8(30)$ & $6(21)$ \\
\hline Tertiary & $28(51)$ & $12(44)$ & $16(57)$ \\
\hline Missing & $3(5)$ & $0(0)$ & $3(11)$ \\
\hline \multicolumn{4}{|c|}{ In receipt of benefits, $\mathrm{n}(\%)$} \\
\hline Yes & $23(42)$ & $10(37)$ & $13(46)$ \\
\hline No & $27(49)$ & $16(59)$ & $11(39)$ \\
\hline Missing & $5(9)$ & $1(4)$ & $4(14)$ \\
\hline
\end{tabular}

\section{Intervention fidelity}

Overall, content (mean 1.65) and time adherence (mean $108 \%$ of the scheduled time) were satisfactory but with inconsistencies. Content adherence was poorest for session 2 ('get moving'; 1.42) and session 4 ('shape up'; 1.08 ), which were the two sessions in which new interactive tasks were introduced. Session 5 ('drop the pressure') had the greatest content adherence score (1.92) but was delivered more quickly than planned $(72 \%$ of scheduled time). In session 7 , the time spent on the education content was double that of the scheduled time (online supplemental table S7).

The educator interviews illuminated some fidelity issues:

- While session 1 was designed to adhere to pedagogic practice in providing scaffolding for the remaining sessions (eg, identifying objectives, goal setting), educators concurred this did not suit the participants, was not easily delivered and the session felt rushed, with corners often cut in content or interactivity: "we probably stuck to the taught curriculum very well and the practical tasks very well, but probably not the goal setting, problem-solving, the tasks for them.... The first session was, the time, we just didn't deliver anywhere near the whole session". Group 1-Lead Educator. This had a knock-on effect on session 2: as one assessor noted elements could not be done because 'probably hadn't had time to give out the task cards [in Session 1]'.

- Educators all agreed that the educational/discussion part of the sessions, was too packed, resulting in some tasks being dropped/covered less well: "The overall programme was excellent. I think my only issue was timing. It was cutting it a bit fine for a lot of them.... if we're trying to encourage them to engage and to give that kind of feedback as to how they've done, or any issues that they might have". Group 4/5-Lead educator.

- Most educators felt they were 'breaking the rules' by dropping or changing aspects but perceived this as necessary to accommodate participant needs: "Some of the educators really went by the book. I guess that's what they're meant to do, but with $X$, they really allowed people to ask questions which are not part of the session. They would spend a lot of time going over those type of things, so it made the class quite interesting, even if we didn't really cover everything". Group 5-Lay educator.

- Practical issues, like equipment availability, also prevented fidelity: "the scales were too heavy to carry around, so they didn't come to the session, so we basically, in this programme, the weight thing got completely left. It just didn't get delivered". Group 1-Lead educator.

\section{Acceptability of the intervention-patient and educator}

Overall, the educator and participant data showed that HEAL-D was acceptable and successfully implemented (online supplemental table S6). Numerous statements illustrated that participants had taken on board the advice and information and made real changes in their life. Concerning the structure and content, participants and educators provided examples of good operationalization and effectiveness in practice. Some delivery had to be matched to individual groups for optimal engagement, such as: the use of videos and PowerPoints versus activities; demonstrating and discussion; the relative inputs of the lay and professional educators.

The acceptability and usefulness of the chosen BCTs were evaluated. Those BCTs focused particularly at improving knowledge and skills were seen to be highly 


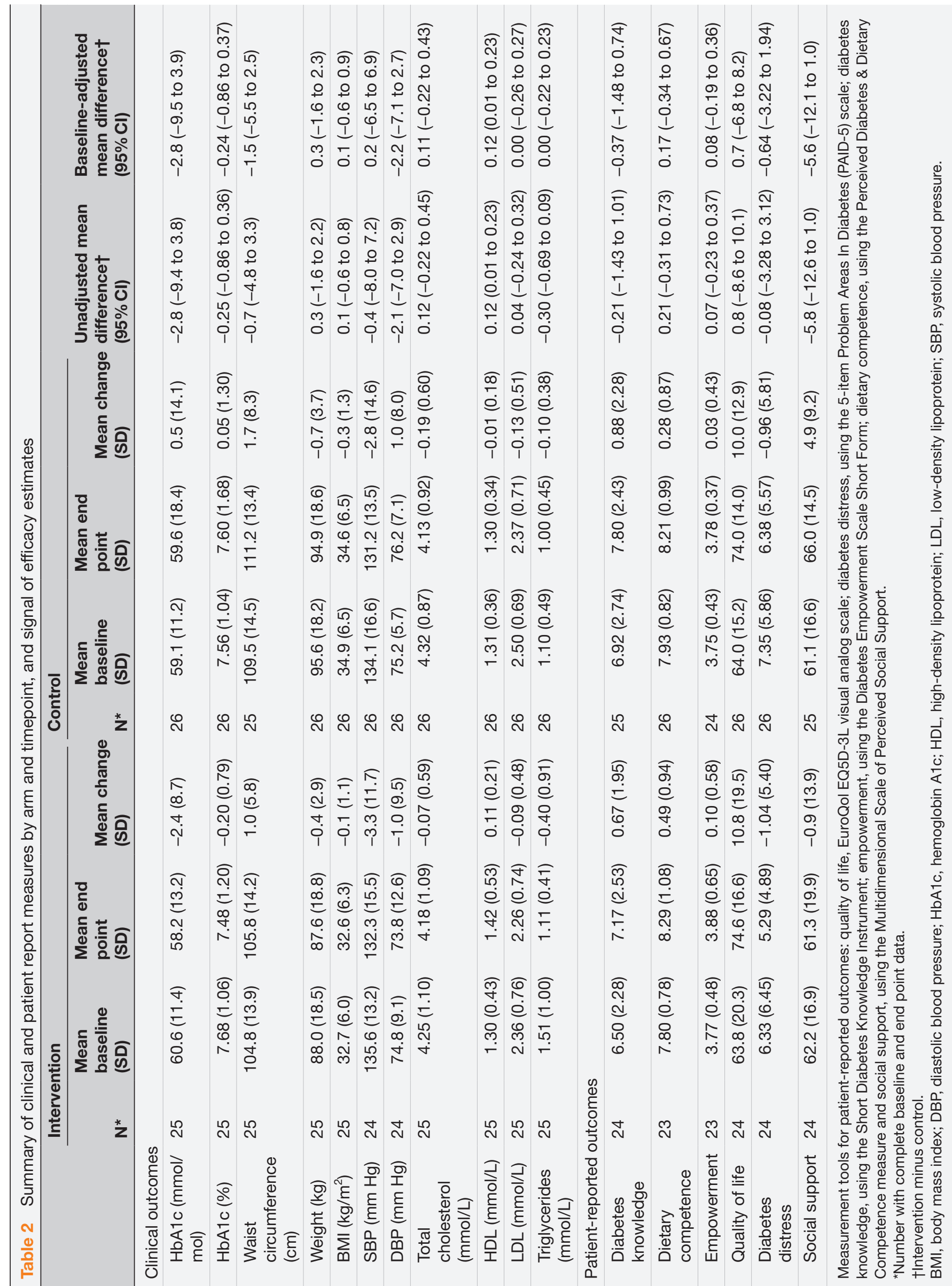


Table 3 Summary of self-reported physical activity levels using the IPAQ questionnaire, by arm and timepoint

\begin{tabular}{|c|c|c|c|c|c|c|}
\hline & \multicolumn{3}{|c|}{ Intervention $\left(n^{*}=23\right)$} & \multicolumn{3}{|c|}{ Control $\left(n^{*}=25\right)$} \\
\hline & Baseline & End point & Change & Baseline & End point & Change \\
\hline \multicolumn{7}{|c|}{ IPAQ activity, n (\%) } \\
\hline Low & $11(48)$ & $6(26)$ & $-5(-22)$ & $10(40)$ & $9(36)$ & $-1(-4)$ \\
\hline Moderate & $10(43)$ & $16(70)$ & $+6(+27)$ & $13(52)$ & $14(56)$ & $+1(+4)$ \\
\hline High & $2(9)$ & $1(4)$ & $-1(-5)$ & $2(8)$ & $2(8)$ & $0(0)$ \\
\hline
\end{tabular}

*Number with complete baseline and end point data.

IPAQ, International Physical Activity Questionnaire.

effective. The balance between theoretical and practical components of demonstrating the behavior were valued (eg, participatory physical activity and cook and taste sessions alongside verbal and visual information). The participatory physical activity sessions and cooking demonstrations seemed to increase both capability and self-efficacy, empowering participants to repeat the activities outside of the sessions. Components designed to achieve social support (eg, group sessions facilitated for interaction and support) and social comparison (eg, sharing experiences) were well received. The group interaction was key as individuals motivated each other and learned from each other, which gave the information credibility and salience that they may not have previously experienced. These components supported the learning by raising self-efficacy, making it acceptable to challenge traditions and created confidence to resist social pressures. The use of credible sources (eg, culturally concordant lay educators and videos with tips from faith leaders and other community members), reinforced the social acceptability of the new behaviors. BCTs aimed at supporting behavior regulation and self-efficacy (eg, self-monitoring, goal-setting, action planning, problem-solving) were less frequently mentioned in the evaluations and appeared to be less effective in supporting behavior change.

Overall, the observations and evaluations identified the need for the following refinements:

- Restructuring of session 1 to focus more on education to provide a foundation for goal-setting. Move goalsetting to session 3,4 or 5 .

- Restructuring of session 7 to allow more time for 'question and answer' so participants conclude the course with needs addressed.

- Ensure availability of equipment storage at venues to enable BCT delivery as planned.

- Modify/Expand educator training to provide more BCT training and ensure that key components of delivery are prioritised and delivered as intended.

- Modify educator training to ensure measurable competence and attainment of required skills set.

\section{DISCUSSION}

Using mixed methods, we have evaluated the acceptability of HEAL-D, an evidence-based, culturally tailored
DSMES program for black-British adults, alongside assessing intervention fidelity and trial feasibility. Importantly, we have shown that it is feasible to recruit, randomize and retain black-British adults with T2D in an RCT, and to implement a culturally tailored DSMES program in primary care, training healthcare professionals and lay educators to deliver a curriculum of evidence-based BCTs. Our attendance data demonstrate, overall, a high degree of acceptability among participants. Our in-depth evaluation methods have enabled us to understand the operationalization of HEAL-D, particularly understanding what elements worked and what components need refinement. This is important in allowing us to refine the delivery to enhance effectiveness and uptake. ${ }^{27-29}$

We engaged key stakeholders, particularly people living with T2D, healthcare professionals and community leaders, in developing HEAL-D to ensure its sensitivity to service users while also being implementable and having adoption potential by the health service. Intervention co-development is time-consuming, requiring a multidisciplinary collaborative approach and is therefore expensive to conduct. ${ }^{30}$ Co-development of HEAL-D was conducted over an 18-month period and involved three phases of qualitative research. ${ }^{17}$ This enabled us to identify priorities, from both a patient and service provider perspective, for the intervention and where there were competing priorities to resolve these with our stakeholders. The HEAL-D program reflects this input, with specific elements that would otherwise not have been chosen if we had used a researcher-driven, 'top down' approach. An example is the flexible attendance schedule, identified as a priority by patients and subsequently used by a third of participants, resulting in near maximal attendance.

The development and evaluation of complex behavioral interventions should include assessment of intervention fidelity, focusing on transferring principles and processes based around theorized mechanisms of change. ${ }^{32}$ While fidelity is a multidimensional construct and there is little consensus about its key elements, recently it has been proposed to consist of five domains: study design; training; intervention delivery; intervention receipt by participants and intervention enactment, 
defined as the extent to which participants apply the skills learned. ${ }^{32-34}$ In the evaluation of HEAL-D, we focused on the complexity of intervention delivery, receipt and enactment, using observations of delivery alongside participant focus groups and educator interviews. The effectiveness of complex interventions is often dependent on the skills of those delivering them. Due to time and resource limitations, we were not able to focus in detail on developing educator training modules and processes for evaluating educator competencies. Our delivery was largely manualized, and our fidelity observations focused on assessing 'adherence', defined as the extent to which the educators delivered the essential content prescribed in the manual. 'Competence' is a related construct, which includes the ability of educators to responsively tailor intervention content and develop a collaborative and trusting relationship with participants as well as accurately pacing delivery and content. ${ }^{35-37}$ We did not systematically assess competence, but our data confirm the need for further training of educators, particularly around the BCTs and appropriate pacing of delivery.

A major strength of our work is our use of mixed methods, enabling a much greater understanding of the delivery and acceptability of our intervention than would be the case from purely quantitative assessments and is increasingly recognised as an area of 'best practice' in feasibility trials. ${ }^{38}$ The sociodemographic profile of our participants shows that we engaged a diverse range of participants. We did not see any clear differences in attendance or acceptability based on gender, socioeconomic status or age. However, we do acknowledge that this trial largely recruited from two South London boroughs in which people of black-British ethnicity form a 'majorityminority' ethnic group, therefore, it was highly relevant to both the local communities and healthcare practitioners. It will be important that a future trial evaluates HEAL-D in different areas/regions and considers issues of implementation where people of black-British ethnicity are not so well represented. We principally focused on two methods of recruitment, primary care database screening with invitation letters and clinic referrals from healthcare practitioners; our recruitment rates are calculated from only the database screening letters of invitation and show a low response rate. While we were not able to formally quantify the response rate to practitioner referrals, anecdotally we observed that this was more effective. This is important for a future trial, in which focusing on recruitment through referral pathways rather than invitation letters is recommended. This feasibility trial employed broad eligibility criteria, avoiding the need for additional screening visits. However, a small number of randomized participants were subsequently found to have high HbAlc, deemed in need of intensive medical management, thus these participants were excluded from the data analysis. A trial will need to have eligibility criteria and screening procedures that ensures the suitability of participants prior to randomization. Our focus group evaluations of intervention acceptability included participants from our co-design study as it was not feasible to run separate groups; these participants may have viewed the intervention differently due to their prior involvement and may have introduced a source of bias in the data. Additionally, our study did not evaluate the feasibility of generating economic data; an evaluation of the cost-effectiveness of the intervention would be an important aspect of a future trial.

In conclusion, culturally appropriate T2D education has been shown to bring about significantly greater benefits for people from minority ethnic backgrounds compared with standard education. ${ }^{15}$ To be effective it is important that interventions are sensitive to the needs of patients, while also being implementable within the healthcare system. We have shown that it is possible to recruit and retain black-British adults with T2D in a trial of a culturally tailored DSMES program, and train healthcare professionals to deliver the intervention in primary care with good fidelity. It will be important to take the HEAL-D intervention forward to a fully powered trial to evaluate its clinical and cost-effectiveness. It is important that we use our qualitative data to recognise and address any potential implementation issues before HEAL-D is rolled out at larger scale.

\section{Author affiliations}

${ }^{1}$ Department of Nutritional Sciences, King's College London, Faculty of Life Sciences and Medicine, London, UK

${ }^{2}$ Social Research Institute, University College London, London, UK

${ }^{3}$ Population Health, King's College London, Faculty of Life Sciences and Medicine, London, UK

Acknowledgements The authors are grateful to the management and staff of the Clinical Research Facility in the NIHR Biomedical Research Centre at Guy's Hospital for their assistance, particularly research nurses Joseline Islam, Salahah Ahmed, Tijani Olawale, Latha Aravindan and Najat Zahira, and physical therapy specialist Eddie Daly for their involvement in the data collection visits. The authors would like to thank Esma Bulut for her assistance with the study administration, the management and staff at the Lambeth Early Intervention and Prevention Service (LEIPS) for their assistance in the physical activity assessments and sessions, and Shola Oladipo at Food For Purpose for the delivery of the Cook \& Taste sessions and Winston Squire for involvement in the physical activity videos. The authors are grateful for the assistance of the Clinical Research Network staff and the Lambeth Intermediate Services team for their assistance with participant recruitment.

Contributors All authors have made substantial contributions to this study. LMG, $\mathrm{CR}$ and $\mathrm{SH}$ were responsible for the conception and design of the study. LMG, CR, $\mathrm{SH}$ and $\mathrm{AM}$ developed the protocol and study approach. LMG and AM undertook data collection. LMG, CR and AM undertook qualitative data analysis. FR and NB-H undertook statistical analysis. LMG drafted the manuscript. All authors read, revised and approved the final manuscript. LMG is guarantor.

Funding This report is independent research arising from a Career Development Fellowship (Louise M. Goff, CDF-2015-08-006) supported by the National Institute for Health Research. LMG and AM are supported by the National Institute for Health Research (NIHR) Applied Research Collaboration South London at King's College Hospital NHS Foundation Trust. FR and NB-H are supported by the NIHR Biomedical Research Centre based at Guy's and St Thomas' NHS Foundation Trust and King's College London.

Disclaimer The views expressed in this publication are those of the author(s) and not necessarily those of the NHS, the National Institute for Health Research or the Department of Health. The funder had no role in the study design, data collection, data analysis, data interpretation or writing of the report.

Competing interests None declared.

Patient consent for publication Not required. 
Ethics approval The study was approved by the London-Fulham Research Ethics Committee (17-L0-1954), and all participants provided written informed consent prior to participation.

Provenance and peer review Not commissioned; externally peer reviewed.

Data availability statement Data are available upon reasonable request. Data are available upon request from the corresponding author.

Supplemental material This content has been supplied by the author(s). It has not been vetted by BMJ Publishing Group Limited (BMJ) and may not have been peer-reviewed. Any opinions or recommendations discussed are solely those of the author(s) and are not endorsed by BMJ. BMJ disclaims all liability and responsibility arising from any reliance placed on the content. Where the content includes any translated material, BMJ does not warrant the accuracy and reliability of the translations (including but not limited to local regulations, clinical guidelines, terminology, drug names and drug dosages), and is not responsible for any error and/or omissions arising from translation and adaptation or otherwise.

Open access This is an open access article distributed in accordance with the Creative Commons Attribution Non Commercial (CC BY-NC 4.0) license, which permits others to distribute, remix, adapt, build upon this work non-commercially, and license their derivative works on different terms, provided the original work is properly cited, appropriate credit is given, any changes made indicated, and the use is non-commercial. See: http://creativecommons.org/licenses/by-nc/4.0/.

ORCID iD

Louise M Goff http://orcid.org/0000-0001-9633-8759

\section{REFERENCES}

1 Pham TM, Carpenter JR, Morris TP, et al. Ethnic differences in the prevalence of type 2 diabetes diagnoses in the UK: cross-sectional analysis of the health improvement network primary care database. Clin Epidemiol 2019;11:1081-8.

2 Becker E, Boreham R, Chaudhury M. Health survey for England 2004. The health of minority ethnic groups. London: Joint Health Surveys Unit, National Centre for Social Research, Department of Epidemiology and Public Health at the Royal Free and University College Medical School, 2006.

3 Lanting LC, Joung IMA, Mackenbach JP, et al. Ethnic differences in mortality, end-stage complications, and quality of care among diabetic patients: a review. Diabetes Care 2005;28:2280-8.

4 James GD, Baker P, Badrick E, et al. Type 2 diabetes: a cohort study of treatment, ethnic and social group influences on glycated haemoglobin. BMJ Open 2012;2. doi:10.1136/ bmjopen-2012-001477. [Epub ahead of print: 1810 2012].

5 Lorig KR, Holman HR. Self-management education: context, definition, and outcomes and mechanisms. Behavioural Science 2000;26:1-17.

6 Clement S. Diabetes self-management education. Diabetes Care 1995;18:1204-14.

7 Sport England. Active lives adult survey may 2019/20 report; 2020.

8 Goff LM, Timbers L, Style H. Dietary intake in black British adults; an observational assessment of nutritional composition and the role of traditional foods in UK caribbean and West African diets. Public Health Nutr 2014:1-11.

9 Vermeire E, Hearnshaw H, Rätsep A, et al. Obstacles to adherence in living with type-2 diabetes: an international qualitative study using meta-ethnography (EUROBSTACLE). Prim Care Diabetes 2007:1:25-33.

10 Wilson C, Alam R, Latif S, et al. Patient access to healthcare services and optimisation of self-management for ethnic minority populations living with diabetes: a systematic review. Health Soc Care Community 2012;20:1-19.

11 Powers MA, Bardsley J, Cypress M, et al. Diabetes selfmanagement education and support in type 2 diabetes. Diabetes Educ 2017;43:40-53.

12 National Institute for Health and Care Excellence. Type 2 diabetes in adults: management (NG28), 2015.

13 Coonrod BA, Betschart J, Harris MI. Frequency and determinants of diabetes patient education among adults in the U.S. population. Diabetes Care 1994;17:852-8.

14 Wierenga ME, Wuerthrich KL. Diabetes program attrition: differences between two cultural groups. Health Val 1995;19:9.
15 Creamer J, Attridge M, Ramsden M, et al. Culturally appropriate health education for type 2 diabetes in ethnic minority groups: an updated cochrane review of randomized controlled trials. Diabet Med 2016;33:169-83.

16 Liu J, Davidson E, Bhopal R, et al. Adapting health promotion interventions to meet the needs of ethnic minority groups: mixedmethods evidence synthesis. Health Technol Assess 2012;16:1-469.

17 Goff LM, Moore AP, Harding S, et al. Development of healthy eating and active lifestyles for diabetes, a culturally tailored diabetes selfmanagement education and support programme for Black-British adults: a participatory research approach. Diabet Med 2021:e14594.

18 Goff LM, Moore AP, Rivas C, et al. Healthy eating and active lifestyles for diabetes (HEAL-D): study protocol for the design and feasibility trial, with process evaluation, of a culturally tailored diabetes self-management programme for African-Caribbean communities. BMJ Open 2019;9:e023733.

19 Moore AP, Rivas CA, Stanton-Fay S, et al. Designing the healthy eating and active lifestyles for diabetes (HEAL-D) self-management and support programme for UK African and caribbean communities: a culturally tailored, complex intervention under-pinned by behaviour change theory. BMC Public Health 2019;19:1146.

20 Samuel-Hodge CD, DeVellis RF, Ammerman A, et al. Reliability and validity of a measure of perceived diabetes and dietary competence in African American women with type 2 diabetes. Diabetes Educ 2002;28:979-88.

21 Craig CL, Marshall AL, Sjöström M, et al. International physical activity questionnaire: 12 -country reliability and validity. Med Sci Sports Exerc 2003;35:1381-95

22 Quandt SA, Ip EH, Kirk JK, et al. Assessment of a short diabetes knowledge instrument for older and minority adults. Diabetes Educ 2014;40:68-76.

23 Anderson RM, Fitzgerald JT, Gruppen LD, et al. The diabetes empowerment scale-short form (DES-SF). Diabetes Care 2003;26:1641-2.

24 Zimet GD, Dahlem NW, Zimet SG, et al. The multidimensional scale of perceived social support. J Pers Assess 1988;52:30-41.

25 Rabin R, de Charro F. EQ-5D: a measure of health status from the euroQol group. Ann Med 2001;33:337-43.

26 McGuire BE, Morrison TG, Hermanns N, et al. Short-form measures of diabetes-related emotional distress: the problem areas in diabetes scale (PAID)-5 and PAID-1. Diabetologia 2010;53:66-9.

27 CECAN) TCftEoCatN. magenta book supplementary guide on handling uncertainty in policy evaluation. HM Treasury 2020

28 O'Cathain A, Croot L, Duncan E, et al. Guidance on how to develop complex interventions to improve health and healthcare. BMJ Open 2019;9:e029954.

29 Hawe P, Shiell A, Riley T. Complex interventions: how "out of control" can a randomised controlled trial be? BMJ 2004;328:1561-3.

30 Jull J, Giles A, Graham ID. Community-based participatory research and integrated knowledge translation: advancing the co-creation of knowledge. Implement Sci 2017;12:150.

31 Duncan E, O'Cathain A, Rousseau N, et al. Guidance for reporting intervention development studies in health research (guided): an evidence-based consensus study. BMJ Open 2020;10:e033516.

32 Bellg AJ, Borrelli B, Resnick B, et al. Enhancing treatment fidelity in health behavior change studies: best practices and recommendations from the NIH behavior change consortium. Health Psychol 2004;23:443-51.

33 Resnick B, Bellg AJ, Borrelli B, et al. Examples of implementation and evaluation of treatment fidelity in the BCC studies: where we are and where we need to go. Ann Behav Med 2005;29 Suppl:46-54.

34 Resnick B, Inguito P, Orwig D, et al. Treatment fidelity in behavior change research: a case example. Nurs Res 2005;54:139-43.

35 Creed TA, Kendall PC. Therapist alliance-building behavior within a cognitive-behavioral treatment for anxiety in youth. $J$ Consult Clin Psychol 2005;73:498-505.

36 Davidson K, Scott J, Schmidt U, et al. Therapist competence and clinical outcome in the prevention of parasuicide by manual assisted cognitive behaviour therapy trial: the POPMACT study. Psychol Med 2004;34:855-63.

37 Lochman JE, Boxmeyer C, Powell N, et al. Dissemination of the coping power program: importance of intensity of counselor training. $J$ Consult Clin Psychol 2009;77:397-409.

38 O'Cathain A, Hoddinott P, Lewin S, et al. Maximising the impact of qualitative research in feasibility studies for randomised controlled trials: guidance for researchers. Pilot Feasibility Stud 2015;1:32. 